\title{
Norois
}

Environnement, aménagement, société

$222 \mid 2012$

Xynthia

\section{Le processus de production du risque « submersion marine » en zone littorale : l'exemple des territoires « Xynthia »}

The production process of flooding risk in coastal area: the example of "Xynthia" areas

Freddy Vinet, Stéphanie Defossez, Tony Rey et Laurent Boissier

\section{OpenEdition \\ Journals}

Édition électronique

URL : http://journals.openedition.org/norois/3834

DOI : $10.4000 /$ norois.3834

ISBN : 978-2-7535-1843-8

ISSN : 1760-8546

Éditeur

Presses universitaires de Rennes

Édition imprimée

Date de publication : 28 février 2012

ISBN : 978-2-7535-1815-5

ISSN : 0029-182X

\section{Référence électronique}

Freddy Vinet, Stéphanie Defossez, Tony Rey et Laurent Boissier, « Le processus de production du risque " submersion marine » en zone littorale : l'exemple des territoires «Xynthia » ", Norois [En ligne], 222 | 2012, mis en ligne le 30 mars 2014, consulté le 31 mars 2021. URL : http:// journals.openedition.org/norois/3834; DOI : https://doi.org/10.4000/norois.3834 

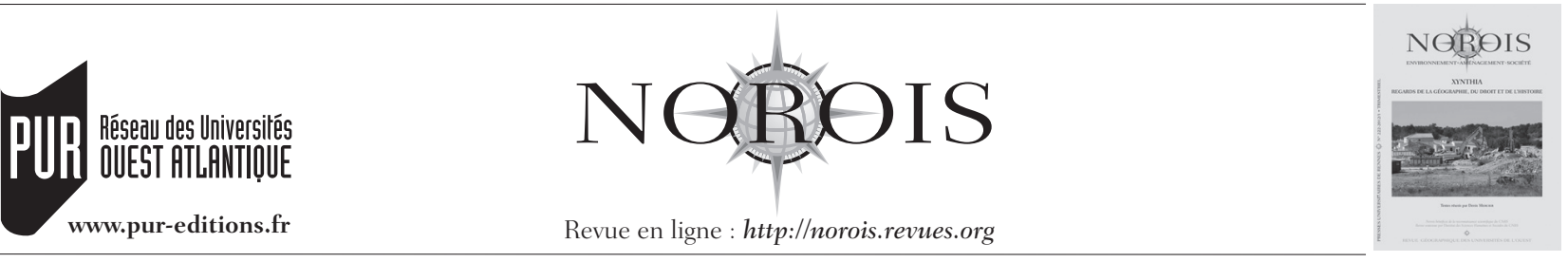

\title{
Le processus de production du risque «submersion marine » en zone littorale : l'exemple des territoires « Xynthia »
}

\author{
The production process of flooding risk in coastal area: the example of "Xynthia" areas
}

\author{
Freddy Vinet*, Stéphanie Defossez, Tony Rey, Laurent Boissier
}

\author{
*Auteur correspondant \\ UMR GRED 0007 CNRS, université Paul-Valéry, Montpellier 3, Route de Mende - 34199 MonTPELLiER \\ Cedex 5, France (freddy.vinet@univ-montp3.fr) (stephanie.defossez@univ-montp3.fr) (tony.rey@univ-montp3.fr) \\ (laurent.boissier@univ-montp3.fr)
}

Résumé : Les lourdes conséquences des catastrophes naturelles restent (trop) souvent expliquées, à travers les médias notamment, par les phénomènes de changements climatiques, par la défaillance des systèmes de défense ou par le caractère exceptionnel des conditions naturelles comme ce fut le cas lors des événements de février 2010 «Xynthia ». Or de tels phénomènes de submersion marine ont déjà atteint le littoral atlantique par le passé. Certes les dommages matériels et humains étaient moindres mais les conditions naturelles similaires. La différence se trouve dans l'occupation des sols bien plus tournée vers l'urbanisation ces 50 dernières années. Notre objectif est de montrer que loin d'être le fruit d'un phénomène exceptionnel, la submersion marine issue de la tempête Xynthia est l'aboutissement d'un processus de production du risque massif et récent. L'étude a concerné les communes de CharenteMaritime et Vendée qui ont subi des pertes humaines. La densification de l'urbanisation et plus généralement l'aménagement du territoire, même s'ils ne sont pas à l'origine du phénomène, ont accru les enjeux toujours plus vulnérables dans les zones à risques, accentuant ainsi les conséquences matérielles et humaines des phénomènes naturels. La tempête Xynthia a généré une submersion marine d'occurrence sans doute rare mais qui rencontre des dynamiques territoriales très rapides. Un espace quasi désert peut se retrouver en trente ans fortement urbanisé. Cet exemple rappelle que les mesures de prévention du risque en particulier les mesures de réglementation de l'occupation des sols doivent s'apprécier sur la longue durée car leur transgression est quasiment irréversible.

\begin{abstract}
The huge aftermaths of disasters remain (too) often explained, through the media, by the climate change or by the failure of flood defenses, as was the storm Xynthia that hit the western part of France in February 2010. However such phenomena of marine flooding linked to windstorm have already struck the Atlantic coast in the past. Material and human damage were lower although natural conditions were quite similar. The difference lies in the urbanization that has widely grown for fifty years. Our goal is to show that the toll of the Xynthia storm is not essentially due to an exceptional marine flooding phenomenon. On the contrary, it is due to the accumulation of vulnerable assets in flood prone zone. The study mainly relies on the seven communes of Charente-Maritime and Vendée which suffered human losses. The densification of urbanization and more generally the lack of land use control led to an anarchic development of assets at risk especially touristic plants and secondary homes. The marine flooding generated by Xynthia Storm has probably a low return period but the low frequency phenomenon has met very rapid territorial dynamics. Thus the process of "production of risk" had been very fast. A quite uninhabited space can be turned into an highly urbanized territory within thirty years. This example reminds that the measures of prevention of risk, especially land use planning must be taken and assessed on the long term because their transgression is irreversible.
\end{abstract}

Mots clés : submersion marine - Xynthia - vulnérabilité - production du risque - tourisme

Keywords : coastal flooding - Xynthia - vulnerability - production of risks - tourism 


\section{INTRODUCTION}

La tempête Xynthia et la submersion marine qu'elle a généré sur les côtes vendéennes et charentaises a suscité de nombreuses études. Ces études arguent de la complexité des causes à l'origine de la catastrophe. Dans le rapport commandité par le ministère de l'Écologie aux différents corps d'inspection (MEEDDM, 2010), peu de place est faite à l'analyse en profondeur des causes de la catastrophe. Laccent est mis sur les causes immédiates en particulier la gestion de crise (16 pages) et la défaillance des digues (30 pages). La vulnérabilité n'est pas évoquée et l'augmentation des enjeux est analysée uniquement au travers de la défaillance des outils de maîtrise de l'occupation des sols. Or la mise en perspective sur les échelles de temps long est un passage indispensable à l'identification des causes profondes des catastrophes naturelles. Nous postulons que la catastrophe porte en elle la signature d'un processus de production du risque qui voit se rencontrer les tendances plus ou moins rapides d'évolution des enjeux humains avec le phénomène naturel quasi instantané et d'occurrence rare. Ainsi, la tempête Xynthia a frappé des territoires littoraux extrêmement mobiles qui ont évolué très rapidement à la fois dans leurs atours naturels et dans leurs composantes humaines. La submersion marine, qui se produit tous les 30 ou 50 ans, s'abat sur un territoire dont la physionomie a complètement changé. Après avoir retracé les grands traits de la mobilité du milieu littoral charentais et sud vendéen depuis quelques siècles, nous verrons comment l'urbanisation, s'invitant dans des terres qui n'étaient pas vouées initialement à l'accueillir, a fabriqué la future catastrophe.

\section{DE L'ESPACE MARITIME À L'ESPACE À RISQUE}

La configuration actuelle des littoraux vendéen et charentais résulte d'une transformation récente du trait de côte (Verger, 2009) sous l'impulsion de facteurs naturels (érosion, dérive littorale, etc.) mais également anthropiques (assèchement, poldérisation, etc.). Deux zones se sont révélées particulièrement exposées lors des événements de février 2010, les littoraux Poitevin et Charentais. La pénétration de l'eau parfois à plusieurs kilomètres à l'intérieur des terres a montré que ces territoires récemment gagnés sur la mer ne sont pas définitivement acquis. La fragilité et le caractère presque amphibie de ces espaces contrastent avec l'urbanisation et l'installation permanente de l'Homme. La submersion fait partie des processus à l'œuvre dans ces secteurs bas et d'autres événements antérieurs à l'épisode Xynthia (Garnier et Surville, 2010) avaient montré la fragilité et la vulnérabilité du littoral.

\section{L'exposition des littoraux charentais et vendéens aux phénomènes de submersion}

Xynthia: de la tempête annoncée

à la submersion marine

Le 27 février 2010, la côte atlantique française s'attend à faire face à une forte tempête. Soixante-dix départements français sont en vigilance météorologique « orange » et quatre en vigilance « rouge » dont la Vendée et la Charente-Maritime. Les événements se déroulent dans la nuit du 27 au 28 février pendant laquelle une conjonction de facteurs (surcote, vents violents, coefficient de marée élevé) aboutit à la submersion du littoral atlantique et particulièrement les côtes vendéennes et charentaises. Les vitesses des vents de sud-ouest atteignent 110 à $120 \mathrm{~km} / \mathrm{h}$ avec des pointes de l'ordre de 140 à 160 km/h sur l'île d'Oléron et l'île de Ré. Même si ces vitesses paraissent élevées, elles restent inférieures à celles enregistrées en décembre 1999 lors des tempêtes Lothar et Martin ou lors de la tempête Klaus en 2009 (plus de $200 \mathrm{~km} / \mathrm{h}$ dans les deux cas). Ce phénomène était prévu et annoncé par les services de Météo-France. En revanche, la submersion marine, tout au moins d'une telle ampleur, ne semblait pas être attendue. Elle résulte, en plus de l'influence des vents, de la concomitance d'un fort coefficient de marée (102) et d'une dépression barométrique à 977 hpa (Chauveau et al., 2011). La surcote atmosphérique et marégraphique a élevé les niveaux marins jusqu'à 4,7 m NGF (Pedreros et al., 2010), avec un maximum dans l'anse de l'Aiguillon et le Pertuis Breton (Anziani, 2010). La submersion marine a causé la mort de 41 personnes auxquelles se sont ajoutés 6 décès liés au vent (arbres arrachés, etc.).

L'événement a été qualifié de rare mais pas exceptionnel (MEEDDM, 2010), le territoire ayant déjà subi des phénomènes similaires. 
La submersion marine : un phénomène récurrent

Le littoral atlantique a dû faire face à de nombreuses reprises au phénomène de submersion marine lors des derniers siècles (Garnier et Surville, 2010). Plus proche de nous, trois événements similaires à ceux de février 2010 ont été identifiés : le 9 janvier 1924, le 16 novembre 1940 et le 17 février 1957 (tableau 1). La dernière catastrophe ancrée dans les esprits reste celle du 26 décembre 1999, la tempête Martin. Cependant, les deux événements (Xynthia et Martin) ne sont pas comparables puisque les dommages causés en 1999 résultaient principalement du vent et marginalement de la submersion marine. Cette mémoire a faussé la perception du possible en 2010 puisqu'un épisode tempétueux fort était attendu mais ni les autorités ni les populations locales n'envisageaient alors une submersion marine majeure.

Quelques jours après l'épisode Xynthia, la presse fait état de submersions marines qui avaient déjà ravagé le littoral, la comparaison avec le 9 janvier 1924 a été immédiate ${ }^{1}$. Appelées «vimer» localement, ces submersions marines présentent la même conjonction de facteurs : surcote marine, fort coefficient de marée et pression atmosphérique basse. Des communes comme Charron ou Châtelaillon-Plage représentaient les lieux les plus touchés, comme en 2010 (figure 1 - planche I). Bien que connus, ces phénomènes sont rares. Leur période de retour à l'échelle locale serait ainsi de l'ordre de 40 à 50 ans. Garnier et Surville (2010) ne recensent qu'une submersion significative sur la période 1950-2010, celle de 1957 (figure 2). Les vimers sont à inscrire dans la longue durée. Exceptionnels si l'on reste dans une fenêtre d'une quarantaine d'années, leur normalité ne s'appréhende qu'à l'échelle séculaire ou pluriséculaire. Or les dommages et surtout les conséquences humaines de l'épisode de 2010 ont été sans précédent. On ne peut expliquer les conséquences dramatiques de la dernière submersion marine subie par le littoral charentais et vendéen qu'en invoquant d'une part la configuration naturelle des lieux qui a évolué au cours du $\mathrm{Xx}^{\mathrm{e}}$ siècle, rendant le territoire plus fragile, et d'autre part, l'anthropisation des milieux qui expose toujours plus d'enjeux plus ou moins vulnérables notamment depuis les années 1960-1970.

\section{Des littoraux fragiles}

Face aux événements des 27 et 28 février 2010, les littoraux poitevin et charentais ont exprimé une forte vulnérabilité conditionnée par des aspects physiques mais aussi par une anthropisation et une artificialisation du système littoral qui avaient pour objectif la conquête du milieu. Leur évolution sur le temps long met en évidence des processus de colmatage naturel rapides sous l'effet des variations du niveau marin et des apports sédimentaires et ce dès la fin

\begin{tabular}{|c|c|c|}
\hline Date de l'événement & $\begin{array}{c}\text { Conditions naturelles (hauteur d'eau, surcote, } \\
\text { vents...) }\end{array}$ & Principales conséquences \\
\hline 9 janvier 1924 & $\begin{array}{c}\text { Hauteur d'eau : } 6,23 \mathrm{~m} \text { à La Rochelle } \\
\text { Coefficient de marée }: 100 \\
\text { Barométrie }: 973 \mathrm{hPa}\end{array}$ & $\begin{array}{c}\text { Routes coupées, caves et maisons inondées aux } \\
\text { Boucholeurs, des ruptures de digues à Châtelaillon- } \\
\text { plage et sur la pointe de l'Aiguillon }\end{array}$ \\
\hline 16 novembre 1940 & $\begin{array}{c}\text { Hauteur d'eau : } 5,91 \mathrm{~m} \text { à La Rochelle } \\
\text { Coefficient de marée }: 91 \\
\text { Barométrie }: 983 \mathrm{hPa} \\
\text { Vitesses des vents }: 120 \mathrm{~km} / \mathrm{h}\end{array}$ & $\begin{array}{c}\text { Dégâts à Châtelaillon-plage, Fouras, 20 brèches } \\
\text { dans les digues de l'île de Ré (surtout aux portes), } \\
\text { submersion entre Esnandes et Charron, rupture de } \\
\text { digue à l'Aiguillon }\end{array}$ \\
\hline 15 février 1957 & $\begin{array}{c}\text { Hauteur d'eau }: 6,65 \mathrm{~m} \text { à La Rochelle } \\
\text { Coefficient de marée }: 115\end{array}$ & $\begin{array}{c}\text { Les communes les plus touchées : Esnandes, Char- } \\
\text { ron, Châtelaillon-Plage; multiples brèches dans les } \\
\text { digues }\end{array}$ \\
\hline 28 février 2010 & $\begin{array}{c}\text { Vitesses des vents : } 150 \mathrm{~km} / \mathrm{h} \\
\text { Hauteur d'eau : } 4,5 \mathrm{~m} \text { à La Rochelle } \\
\text { Coefficient de marée }: 102 \\
\text { Vitesse des vents : } 130 \mathrm{~km} / \mathrm{h}\end{array}$ & $\begin{array}{c}\text { La Faute-sur-Mer, Charron, Châtelaillon-plage, île } \\
\text { de Ré, brèches et ruptures de digues }\end{array}$ \\
\hline
\end{tabular}

Tableau 1 : Submersions marines sur les littoraux charentais et vendéens au cours du xx siècle (Source : Musereau, 2009; Garnier et Surville, 2010 ; Verger, 2009; préfecture de Charente; Sud-Ouest, 16 février 1957; conservatoire de l'estuaire de la Gironde) Coastal flooding on coast of Charente and Vendée during the $20^{\text {th }}$ century

1. Ouest-France, édition du 4 mars 2010. 
de la transgression marine (André, 1986; Gruet et Sauriau, 1994; Pawlowski, 1998). Mais ce n'est qu'à partir de la conquête des terres agricoles amorcée à la fin du Moyen Âge que s'opèrent les plus grands changements du trait de côte. Cette évolution pluri-séculaire s'exprime aussi dans l'évolution dynamique des flèches littorales qui sous l'influence des variations hydro-sédimentaires et des conditions météomarines s'allongent, s'engraissent, se stabilisent, s'érodent, etc., une dynamique naturelle propice à la résilience des milieux. L'absence de prise en compte de ce temps « géomorphologique » pose problème dans le sens où l'artificialisation récente de ces milieux littoraux a masqué leur dynamique et leur fragilité face à la tempête marine Xynthia.

\section{Le marais Poitevin}

Ce marais maritime de $1200 \mathrm{~km}^{2}$ est bordé par des côtes basses sableuses telles les pointes de l'Aiguillon et d'Arcay en appui sur d'anciennes formations calcaires et des côtes vaseuses caractérisées par l'estuaire du Lay et l'anse de l'Aiguillon. Quatre unités se dégagent donc de ce marais (figure la - planche I). Quelques buttes fossiles (Grues, Saint-Michel-enl'Herm, Triaize, Charron), reliques d'anciennes îles calcaires et à rattacher à la plaine vendéenne, s'inscrivent dans ce marais (Pawlowski, 1998).

L'anse de l'Aiguillon relève d'un remblaiement artificiel très actif à partir du milieu du XVII ${ }^{\mathrm{e}}$ siècle (figure la - planche I). Ce remblaiement sédimentaire s'effectue par les hommes qui drainent et assainissent le marais par des canaux de drainage. La progression du rivage est inégale, maximale au nordouest à l'abri de la flèche de l'Aiguillon, décroissante vers le nord-est puis vers l'est pour s'arrêter au sud (Verger, 2009). La planéité et la faible altitude de la plaine littorale l'exposent de manière certaine aux aléas d'inondation fluviale et de surcote marine.

Côté mer, la flèche de l'Aiguillon, large de 700 mètres et longue de $13 \mathrm{~km}$, peut participer à la protection des marais mais l'érosion l'affecte. Les sables se déposent plus en amont sur la pointe d'Arcay, quant aux alluvions du Lay elles ne parviennent plus sur la flèche littorale. Certaines portions de plages de l'Aiguillon sont alors équipées d'enrochements disposés en épis, ils complètent la Digue de Défense édifiée en 1863. La flèche littorale possède encore quelques dunes dont l'amplitude modérée n'a pas suffi à contenir les assauts des vagues et du vent : une vaste brèche marine s'est formée le 28 février 2010 à l'extrémité sud de la pointe de l'Aiguillon inondant les derniers polders conquis en 1965.

Plus au nord, la flèche d'Arcay, orientée nord-ouest sud-est, longue de $9 \mathrm{~km}$ et large de 900 mètres, se caractérise dans sa partie distale par des plages et des dunes sableuses. Les cartes historiques de Rogier en $1579^{2}$ et La Favolière en $1677^{3}$ dévoilent l'évolution dynamique de la flèche littorale et la déviation de l'embouchure du Lay vers l'est entre 1579-1677. Cette migration continue de l'embouchure provoque une sous-alimentation en sables des plages et des dunes plus à l'ouest. Il en résulte un démaigrissement des anciens cordons littoraux situés entre La Fautesur-Mer et La Tranche-sur-Mer ${ }^{4}$. Par conséquent, la partie proximale de la flèche d'Arcay qui souffre d'un déficit sédimentaire au profit de la partie distale en progradation offre une plus grande vulnérabilité géomorphologique face aux aléas recul du trait de côte et submersion marine.

L'estuaire du Lay s'ouvre entre ces deux flèches littorales. De part et d'autre de ses rives, entre les anciens schorres et les flèches littorales fossiles se sont développées les communes de L'Aiguillon-surMer et de La Faute-sur-Mer. Jusqu'au XvI ${ }^{\mathrm{e}}$ siècle, le fleuve se jetait à la mer à l'est des Roullières (lieuxdits « la Belle-Henriette») puis sous l'impulsion de la formation de la flèche d'Arcay, le tracé fluvial a changé et l'embouchure s'oriente désormais vers le sud-est. L'estuaire semble alors bien plus influencé par la dynamique marine que fluviale. Son envasement y est très important notamment depuis la construction des barrages Braud et de Moricq et aussi en raison de l'extension de la flèche de l'Aiguillon qui confine l'embouchure (figure 1 - planche I).

\section{Les marais charentais}

Les marais charentais se divisent en trois ensembles bien individualisés, le marais septentrional situé au nord de Rochefort, le marais de Brouage et de la Seudre, respectivement au sud de Rochefort et dans la plaine de la Seudre (figure $1 \mathrm{~b}$ - planche I). Les trois marais forment une large plaine basse, avec une cote altitudinale en dessous du niveau des plus hautes mers (4 m NGF). Les marais sont drainés par

2. Rogier P., 1579. Carte du golfe de Poitou au Xvi ${ }^{e}$ siècle.

3. La Favolière, 1677. Carte des Coste depuis les Sables d'Olône jusqu'à Cartignac en Médoc.

4. Les plages orientales de La Tranche-sur-Mer sont aujourd'hui équipées en ouvrages de défenses. 
un réseau de canaux et de fossés reliés pour chacun de ces trois marais aux cours de la Charente, au havre de Brouage et à la Seudre. L'espace gagné sur la mer et le schorre découle d'une démarche historique de grande ampleur (figure 1 - planche I). D'imposants travaux d'assèchement des marais et de poldérisation ont été entrepris dès le XVIII ${ }^{\text {e }}$ siècle (Regrain, 1980). Le colmatage des marais est variable, il est plus rapide pour le marais de Brouage qui ne bénéficie pas de cours d'eau permanent et suffisamment puissant pour évacuer les dépôts vaseux. À l'inverse, la Charente et la Seudre protègent les marais de Rochefort et de la Seudre du colmatage, leurs embouchures restent actives et ne semblent pas subir de grandes modifications morphologiques (Weber, 2004). L'activité se concentre autour de l'ostréiculture. L'espace interdital est occupé par d'anciens parcs à huitres qui cohabitent avec des espaces toujours exploités (Gouleau et al., 2000). Les marais salants en déclin disparaissent progressivement du paysage, ils sont transformés en bassins ostréicoles.

Les littoraux poitevin et charentais se caractérisent donc par leur relative jeunesse et une forte mobilité qui s'expliquent par l'existence de forçages naturels (colmatage de l'anse de l'Aiguillon et de l'estuaire du Lay, évolution cyclique des flèches littorales, Petit Âge Glaciaire) et des pressions humaines depuis la fin du Moyen Âge où l'ère du drainage et de l'assainissement a progressivement laissé la place au temps des endiguements et des enrochements. De par ses caractéristiques, cet espace littoral révèle une réelle vulnérabilité géomorphologique accentuée par l'anthropisation et l'artificialisation des milieux.

Plusieurs communes littorales comme La Tranchesur-Mer, La Faute-sur-Mer, L'Aiguillon-sur-Mer, Charron, etc., occupent d'anciennes îles et cordons littoraux. Leur extension récente occupe des zones basses gagnées naturellement sur la mer ou acquises par d'importants travaux hydrauliques et de constructions de digues. Plusieurs zones d'habitat occupent aujourd'hui des espaces potentiellement très dangereux : rives du Lay, schorres consolidés, polders, dunes fossiles arasées, dépressions inter-dunaires, anciens chenaux maritimes et paléoméandres transformés en marais, etc., des localisations qui semblent faire fi de la possibilité d'une catastrophe naturelle. Pourtant, la dangerosité des agents maritimes (vagues et surcotes) et/ou fluviaux (surverse, brèche) est réelle sur le littoral Poitevin (Raison, 2008) tout comme la possibilité qu'ont certains milieux fossiles à se réactiver au cours d'une submersion marine et/ou d'une crue. L'inondation découle de ce contexte géomorphologique mais aussi d'une fragilité structurelle des levées et des digues.

\section{L'endiguement des littoraux poitevins et charentais}

Nous pouvons distinguer deux grands types de digues, celles édifiées (levées, moellons, digues empierrées) pour gagner des terres agricoles (polders de l'anse de l'Aiguillon et des marais charentais) et non pour protéger des zones urbaines et celles construites pour la protection des plages, des biens et des personnes sur le front de mer (épis, enrochements, perrés, etc.) et sur le long du Lay (digues fluviales). Cette distinction se retrouve dans leurs caractéristiques (taille, matériaux, pente) et leurs objectifs.

La construction des polders a nécessité la construction de plusieurs générations de digues, les plus anciennes apparaissant au XVII ${ }^{\mathrm{e}}$ siècle (figure 2). Quant aux dernières, elles datent de 1965 pour l'anse de l'Aiguillon (digue du Maroc) et 1969 pour les marais charentais (Verger, 2009). En plus de trois siècles, le mode de construction des digues s'est perfectionné. Les anciennes levées (qui restent privées) sont majoritairement composées de bri et de pierres. Jusqu'au XvIII ${ }^{\mathrm{e}}$ siècle, elles sont construites et entretenues par les sauniers et les fermiers exploitants les marais. Ces levées n'ont qu'un rôle secondaire dans la protection contre les inondations. Ensuite et ce jusqu'à la première moitié du XIX siècle se développe la digue édifiée avec des pierres sèches posées sur le bri; l'État a en charge leur construction. La seconde moitié du XIX ${ }^{\mathrm{e}}$ siècle voit l'utilisation du mortier (à base de chaux) et du béton, ce matériau renforce la cohésion des pierres des ouvrages en maçonnerie. Au cours de cette période, les communes se substituent progressivement à l'État. Enfin, le $\mathrm{xx}^{\mathrm{e}}$ siècle est celui de la digue en ciment, matériau artificiel plus polyvalent. Au cours de la tempête du 28 février 2010, quasiment tous les polders du marais poitevin et charentais ont été inondés à partir de surverses et de brèches de débordement. L'inondation a été facilitée par plusieurs facteurs : une cote d'arasement pour les levées et les digues les plus anciennes situées à une vingtaine de centimètres sous le niveau marin actuel (Verger, 2010), des matériaux hétérogènes et de faible résistance et le mauvais état de certaines digues consécutif à un manque d'entretien (Anziani, 


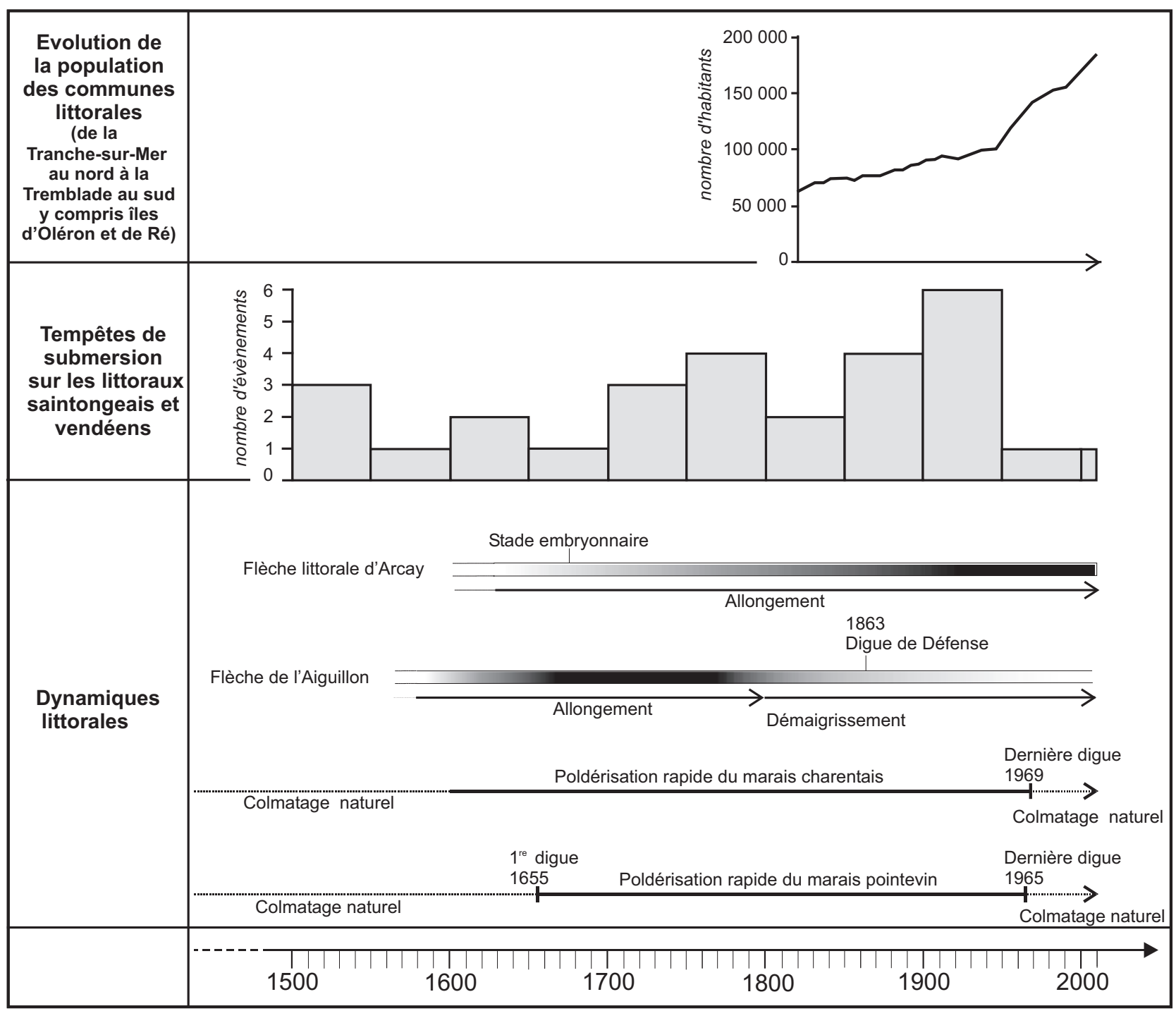

Figure 2 : Temps naturel et temps anthropique sur la côte centre atlantique française (Source : Allard et al., 2008; Verger, 2009; Garnier et Surville, 2010; INSEE (RGP).

Long term evolution of environment and short term evolution of human assets on the French Atlantic Coast

2010). Aujourd'hui encore il subsiste un problème de gouvernance dans l'entretien des digues compte tenu de la pluralité des acteurs (MEEDDM, 2010).

Pour protéger des inondations les communes de La Faute-sur-Mer et de L'Aiguillon-sur-Mer, le Lay a été endigué et ce dès les grandes tempêtes de 1926 et 1929. Certains segments de digues sont vieillissants (Raison, 2008). Alors que les digues en terre ont majoritairement cédé, certaines digues comme celles situées face au camping municipal de La Faute-surMer n’ont pas cédé mais furent « uniquement» submergées en raison des travaux de rehaussement et de renforcement (gabions et matelas RENO) exécutés en 2004. La digue a ainsi participé à la production du risque en favorisant le sentiment de protection des riverains et ce d'autant plus que la plupart des digues n'étaient pas destinées à accueillir des zones urbaines à l'arrière mais des terres agricoles.

Les conséquences des événements de février 2010 sur le milieu naturel

Les phénomènes récurrents de recul de trait de côte et de submersion marine (Raison, 2008; Verger, 2010) trouvent leur écho au cours de la tempête de 2010, les submersions se produisant soit naturellement dans les zones fragilisées du cordon dunaire 
(brèche marine de la Belle Henriette à La Faute-surMer), soit sous le contrôle des nombreux chemins d'accès à la mer. Les dépressions inter-dunaires et les espaces où ont été arasées les dunes ainsi que le linéaire de l'ancienne lagune de la Belle Henriette (Bouhier, 1957) ont été ennoyées; en revanche, les crochets multiples de la flèche sont restés hors d'eau. Les impacts géomorphologiques de la tempête Xynthia sur les cordons littoraux sont considérables. Le déferlement des vagues sur les dunes et le vent ont provoqué un recul moyen du trait de côte estimé entre 3 et 5 mètres, une moyenne qui masque une érosion parfois plus intense comme la plage du Moulin Brûlé sur l'île de Ré qui a enregistré un recul de $22 \mathrm{~m}$ (Pedreros et al., 2010). Ces forçages naturels ont diminué la largeur et la hauteur des dunes, créé des falaises dunaires, accentué le profil des pentes, formé des brèches marines, etc. Les affouillements produits sur le front dunaire et sous les fondations des maisons ancrées dans les dunes ont endommagé certaines habitations situées en bord de mer (La Tranche-sur-Mer). L'érosion du littoral concourt activement à la fragilisation du rempart dunaire dont le niveau de vulnérabilité futur dépendra de sa capacité de résilience post-tempête.

Les ouvrages de protection édifiés le long du Lay et autour des marais ont présenté une efficacité toute relative face à des événements extrêmes : une trentaine d'hectares de terres agricoles en rive gauche avaient déjà été inondés en 1999. Au cours de la tempête Xynthia les vagues et les courants ont remonté et inondé les hauts schorres de l'estuaire s'ajoutant au débit du Lay. Une partie du lit majeur surtout en rive droite où est située La Faute-sur-Mer a été fortement inondée : l'eau s'est engouffrée dans les points bas caractéristiques des anciens schorres, chenaux maritimes et méandres abandonnés, qui le temps de la tempête ont été « réactivés » et facilement immergés. En dépit de cette catastrophe, l'événement aurait pu prendre une toute autre ampleur si à la surcote marégraphique de 2010 s'était ajoutée une crue du Lay. Dans ces conditions, l'estuaire constitue l'espace le plus vulnérable de cette partie du littoral vendéen.

En dépit des ouvrages présents sur le trait de côte, l'exposition du territoire face à l'aléa submersion marine est réelle et historique (Raison, 2008). Ces milieux encore peu stabilisés ont récemment accueilli des populations toujours plus nombreuses et ont subi une densification de l'urbanisation. Ainsi les transfor- mations du milieu littoral impactent directement les sociétés qui s'implantent sur des territoires fragiles et exposés aux risques.

\section{De L'ESPACE NATUREL À L'ESPACE URBANISÉ ET TOURISTIQUE}

La submersion marine "Xynthia » a engendré la mort de 41 personnes endeuillant 7 communes sur les deux départements de la Vendée et CharenteMaritime. L'étude et la localisation précise des victimes (Vinet et al., 2011) tendent à montrer que les décès ont eu lieu dans des secteurs récemment urbanisés. Nous avons voulu prolonger cette réflexion en décrivant les processus qui ont conduit à cette urbanisation massive en zone à risque. Les sept communes de La Faute-sur-Mer (Vendée), Aytré, Esnandes, Charron, Châtelaillon-Plage, La Flotte-en-Ré et Saint-Georges-d'Oléron (Charente-Maritime) nous ont servi d'échantillon d'étude. Elles ont connu depuis les années 1960 une forte croissance démographique. Les deux phénomènes en cause sont la périurbanisation et l'urbanisation littorale (résidences secondaires).

Deux types de communes ont en effet pu être mis en évidence. Leurs activités économiques prédisposent et conditionnent les caractéristiques des populations résidentes en termes socio-professionnel et démographique. Le premier rassemble des communes en voie de périurbanisation où les activités traditionnelles (pêche, agriculture, etc.) cohabitent avec les pendulaires. La part d'employés ne travaillant pas dans la commune (un quart de la population active) pour Aytré, Charron et Esnandes est révélatrice de leur caractère périurbain, surtout Aytré véritable extension urbaine de La Rochelle développée grâce au secteur de la construction. Les trois communes poursuivent leurs activités primaires, la conchyliculture pour Aytré et la mytiliculture à Charron et Esnandes. Elles accueillent en outre une population retraitée $(23 \%)$.

Le second caractérise les communes dont l'activité est tournée vers le tourisme et les résidences secondaires. L'activité principale des communes de La Flotte-en-Ré, Saint-Georges-d'Oléron et La Fautesur-Mer et Châtelaillon-Plage reste tournée vers le tourisme et l'accueil de populations non permanentes depuis les années 1960-1970. En plus de l'attractivité balnéaire, ces communes attirent une forte pro- 
portion de retraités qui viennent s’installer de façon permanente sur le littoral, un tiers de la population communale à La Flotte et Châtelaillon-Plage, un peu moins de la moitié (40\% environ) à Saint-Georgesd'Oléron et La Faute-sur-Mer. Cette part non négligeable de retraités a une incidence certaine sur la pyramide des âges et rend la population de ces communes plus vulnérable encore.

\section{De petites communes en expansion démographique}

Sur l'ensemble des communes étudiées, le nombre d'habitants oscille entre 1001 à La Faute-sur-Mer et 8806 à Aytré en 2007. Il s'agit de communes littorales touristiques et de résidences secondaires et/ou périurbaines, ces dernières attirant la population de La Rochelle (figure 3).
Ces cinquante dernières années ont été marquées par l'augmentation de la population. Saint-Georgesd'Oléron a suivi une croissance régulière de 37,9\% sur la période 1962-2007 proche de la moyenne nationale estimée à $38 \%{ }^{5}$. Seule la commune de Châtelaillon-Plage (13,9\%) a connu une croissance faible tandis que Charron, Aytré, La Flotte-en-Ré, La Faute-sur-Mer sont passées par une véritable explosion démographique doublant ou triplant leur population (Esnandes : $+179 \%$ ) (figure 4).

Cette croissance est quasi continue depuis 1968 malgré une légère baisse dans les années 1990 pour les communes de Châtelaillon-Plage et Aytré concurrencées par l'attraction d'autres communes. La fin des années 1970 et le début des années 1980 représentent un tournant décisif dans la croissance. L'évolution démographique s'explique par les soldes naturel et/ou migratoire selon les communes. La croissance d'Aytré, Charron et Esnandes est fondée

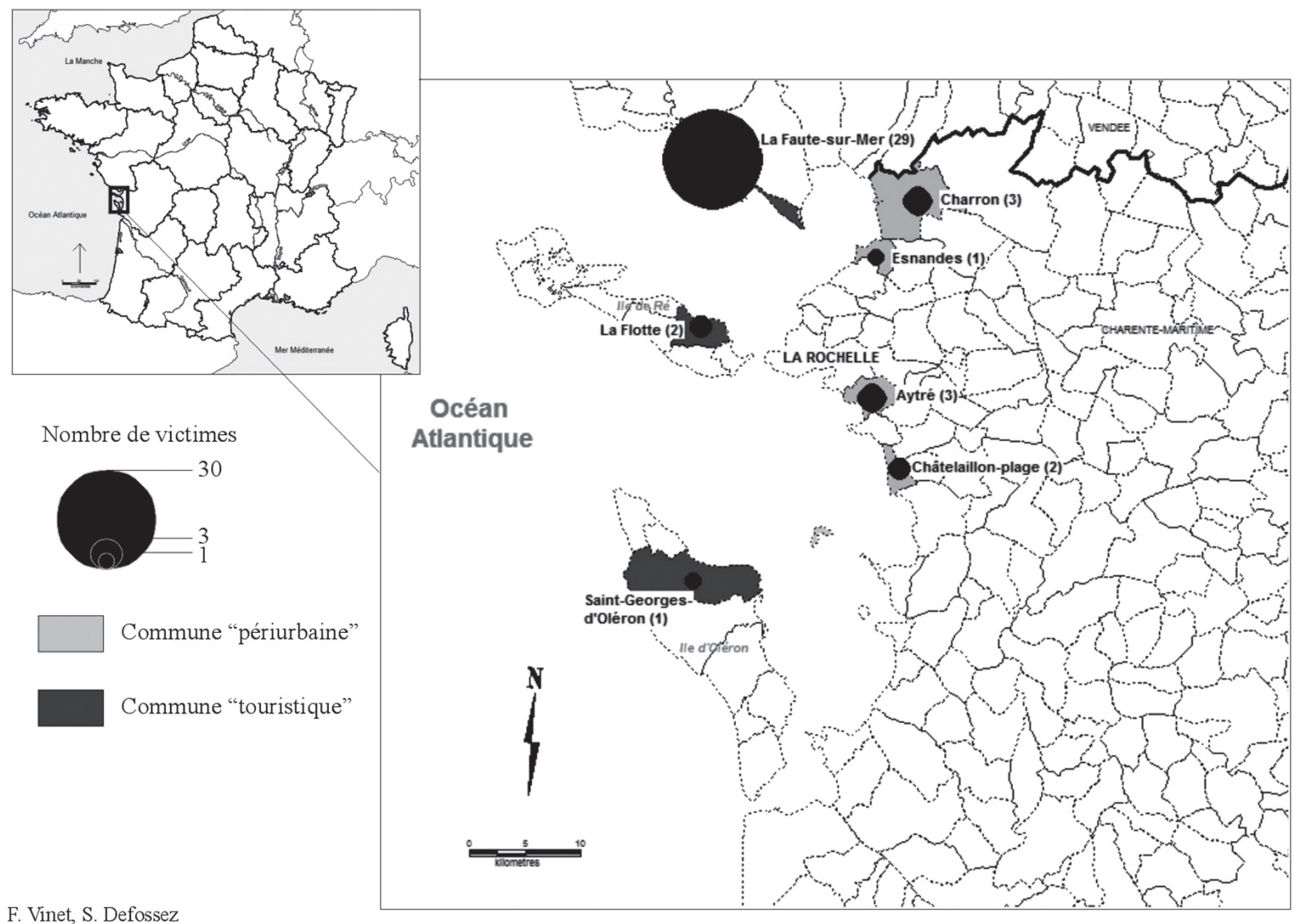

F. Vinet, S. Defossez

Figure 3 : Communes où ont été recensés des décès dus à la submersion marine

Communes with coastal flooding related fatalities

5. Source : INSEE, RGP 1962-2007. 


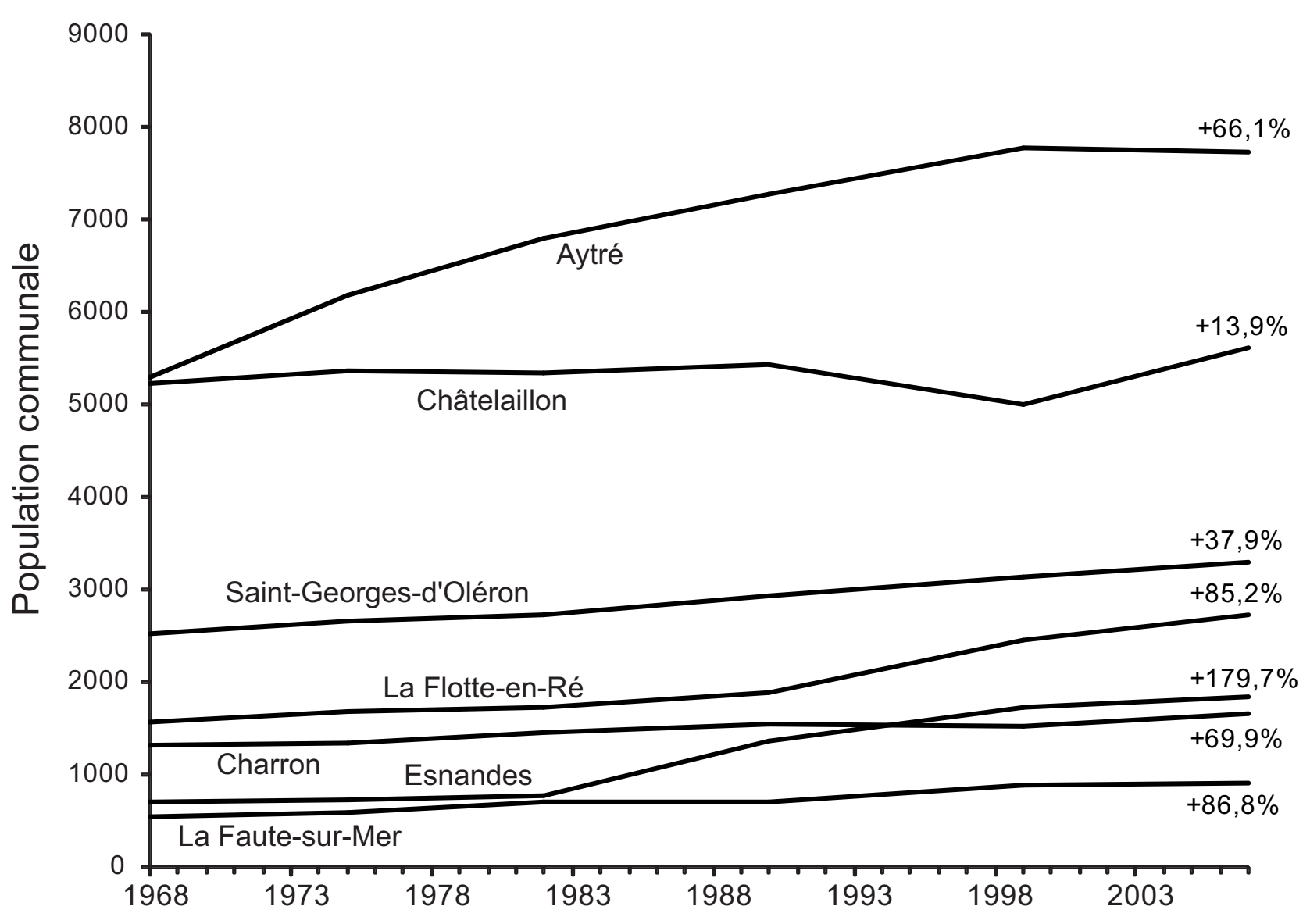

Figure 4 : Évolution de la population des sept communes (concernées par des décès liés à la submersion) entre 1968 et 2007 (source : INSEE - RGP)

Evolution of the population in the seven cities concerned by fatalities (1962-2007)

sur des taux de natalité élevés jusque dans les années 1990 puis l'arrivée de nouveaux urbains. Ces nouvelles implantations s'expliquent principalement par la saturation de la ville de La Rochelle qui déploie son aire d'attractivité vers les communes alentours, faisant évoluer leur statut de communes rurales à communes périurbaines. Notons qu’à Esnandes cette périurbanisation a débuté dès 1975 (solde migratoire de $+7,5 \%$ entre 1975 et $1982^{6}$ ) avec une volonté de densification (Rousseaux, 2009) tandis que les autres communes bénéficient de l'aire d'influence de La Rochelle depuis les années 1990.

Cette augmentation de la population a une incidence sur la demande foncière et immobilière et conditionne l'extension de l'urbanisation dans ces

6. Source : Institut National des Statistiques et des Études économiques : INSEE (base de données locales) [http://www.insee.fr/fr/bases-de-donnees/ default.asp? page=statistiques-locales. $\mathrm{htm}$ ]. zones littorales. Les secteurs proches de la mer occupés par l'agriculture sous la forme de « conches » - petites parcelles agricoles protégées dans les dunes - ou voués à l'élevage pour les terrains marécageux au statut de « communaux » ont laissés leur place à l'urbanisation. Renard (1972) a d'ailleurs bien montré comment le morcellement de la propriété foncière a été, dans les communes littorales du sudVendée, un facteur d'urbanisation rapide.

L'évolution du nombre d'habitants ne rend pas compte du processus de production du risque dans son ensemble puisque les statistiques de population ne concernent que la population permanente. En effet le nombre d'habitants masque la réalité de l'occupation des communes à certaines périodes, particulièrement les périodes de vacances scolaires. Dans les communes touristiques, la quantification des enjeux exposés par l'étude du nombre de logements 
principaux et secondaires et de leurs poids relatifs permet d'avoir une mesure plus sûre de l'augmentation des enjeux.

\section{L'urbanisation du littoral et le développement du tourisme : l'évolution du bâti}

L'évolution de l'urbanisation du littoral ne tient pas seulement au nombre de résidences permanentes dépendantes du nombre d'habitants mais aussi aux résidences secondaires.

Charron, Esnandes et Aytré s'apparentent à des communes périurbaines et peu marquées par le tourisme ou l'occupation non permanente, les résidences secondaires n'y représentant que moins de $10 \%^{7}$ des logements (figure 5). Ces proportions ont peu évolué depuis les années 1960. En revanche, les quatre autres communes montrent un véritable attrait pour le tourisme résidentiel avec $34 \%$ de résidences secondaires à Châtelaillon-Plage, 61 \% à la Flotteen-Ré, $81 \%$ à Saint-Georges-d'Oléron et $87 \%$ pour La Faute-sur-Mer. Cette attractivité touristique et de résidents non permanents s'est développée depuis les années 1960-1970. Dès le recensement de 1968, La Faute-sur-Mer affiche près de $80 \%$ d'habitations en résidences secondaires. À ce type de bâti « en dur » s'ajoutent les quelque 7800 emplacements de campings et 559 chambres d'hôtels dans des établissements classés pour l'ensemble des sept communes étudiées.
La prise en compte de ces capacités d'hébergement importantes laisse augurer des bilans plus graves si les circonstances étaient autres. En effet, le nombre de morts aurait pu être bien plus élevé si un événement similaire à Xynthia avait eu lieu pendant une période de l'année où le taux d'occupation est au maximum.

L'urbanisation et la touristification du littoral ont considérablement développé les enjeux. L'occupation permanente ou temporaire du littoral par des populations nouvelles moins connoté étrangers/locaux a accru la vulnérabilité des territoires littoraux face au risque de submersion.

\section{Des populations âgées et vulnérables}

Comme le rappelait Jean Renard après la catastrophe ${ }^{8}$, les communes du sud-Vendée accueillent des populations qui, touristes saisonniers pendant de nombreuses années, s'installent définitivement à l'âge de la retraite dans les communes comme la Tranche-sur-Mer ou La Faute-sur-Mer. La pyramide des âges (figure 6) et l'âge médian de la population de La Faute-sur-Mer (52 ans contre une moyenne nationale de 38 ans) font de cette commune la caricature des communes littorales qui ont vu l'afflux massif de populations âgées ces dernières décennies. Ces populations âgées sont particulièrement vulnérables comme l'a montré l'étude des personnes décédées lors de la submersion marine du 28 février 2010 (moyenne d'âge 75 ans voir Vinet et al., 2011).

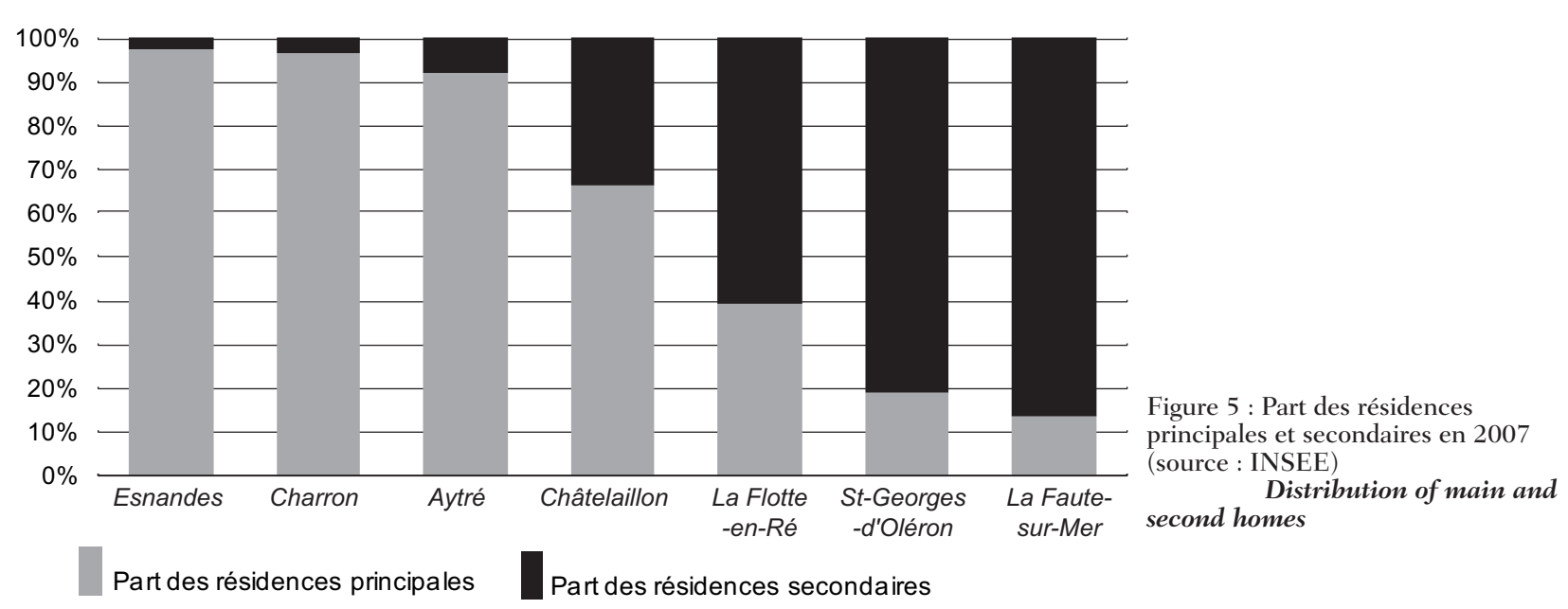

7. Sont exclus dans ce calcul les logements vacants, la part est donc calculée sur la somme des résidences principales et secondaires/occasionnelles.

8. Interview dans Le Monde du 3 mars 2010 et dans La Croix 8 mars 2010. 

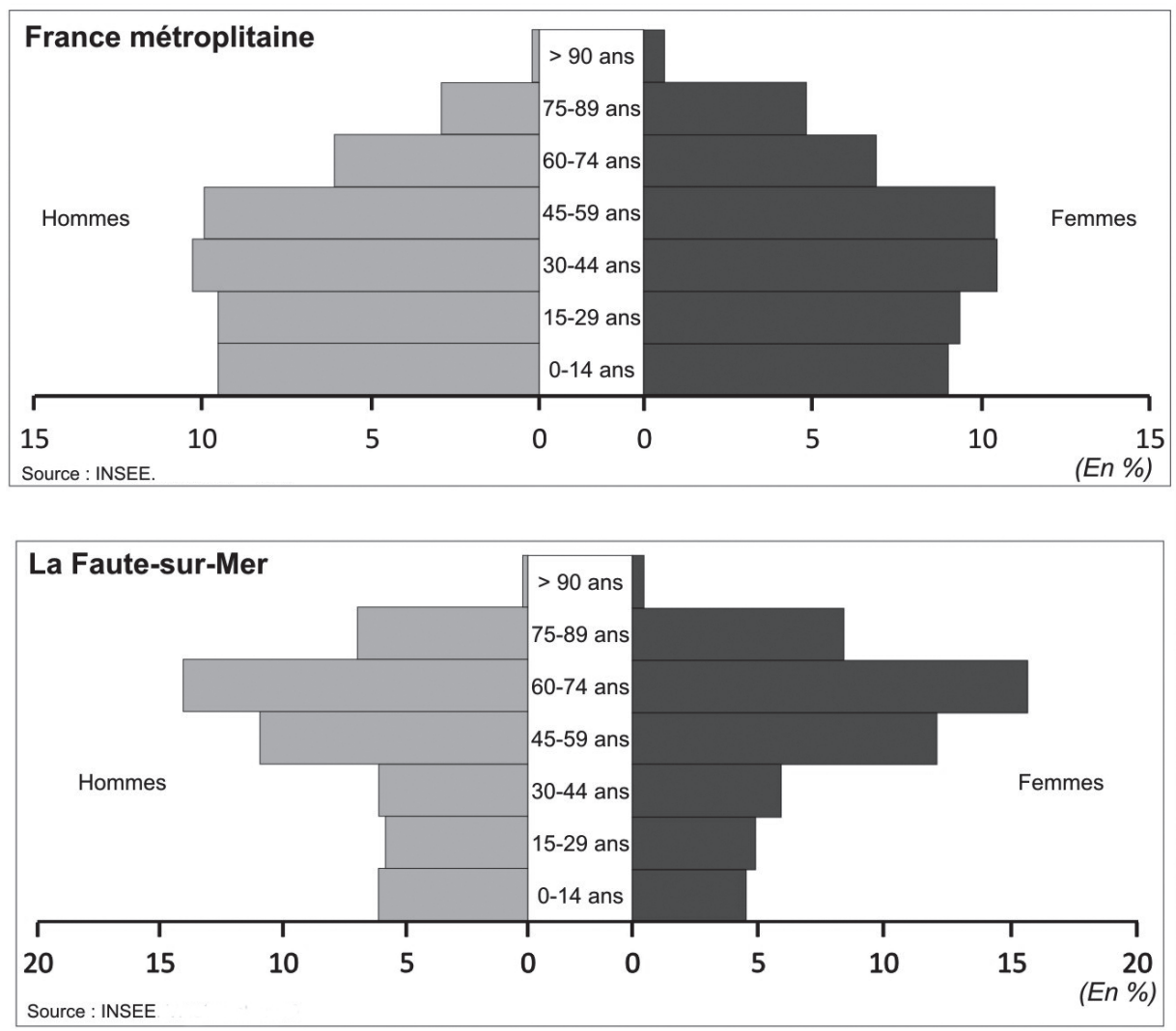

Figure 6 : Pyramide des âges de La Faute-sur-Mer et de la France métropolitaine

Population pyramids of the commune of "La Fautesur-Mer" and of whole France

\section{Le PRocessus de PROduCtion DU RISQUE : L'EXEMPLE dE LA FAUTE-SUR-MER}

La commune de La Faute-sur-Mer représente un véritable cas d'école dans ce processus de production du risque avec une croissance démographique qui induit non seulement une croissance des enjeux mais aussi des vulnérabilités humaines (population âgée) et matérielles (architecture inadaptée). L'évolution de l'urbanisation a été retracée depuis les années 1950 grâce à l'étude diachronique des données cadastrales et démographiques. L'élaboration d'une typologie du bâti appliquée aux zones « noires » a permis d'établir bâti par bâti la vulnérabilité potentielle des occupants face à l'inondation.

\section{La « colonisation » de la flêche d'Arçay}

L'urbanisation et l'occupation des terres littorales par les activités et le logement touristiques ont été décrites dès les années 1970 entre autres par les géographes nantais. Les problèmes pointés alors étaient nombreux sans toutefois que ne soient mis en exergue les menaces d'origine naturelle. Sur des communes du sud-Vendée, Renard (1976) a bien mis en évidence les relations entre structures foncières et développement de l'urbanisation littorale. Il pointe également les conflits et problèmes que pose cette urbanisation massive (conflits entre activités traditionnelles et tourisme, problèmes de pollution et de dégradation des milieux, etc.). Il n'est toutefois pas fait état à l'époque des dangers de submersion marine dans les zones basses littorales. À La Fautesur-Mer, Cabanne (1977) a décrit la transformation du hameau en bourg par la fonction balnéaire. Ce mouvement initié entre les deux guerres s'est amplifié après la création de la commune en 1953 et s'est poursuivi jusqu'à nos jours (Chadenas et al., 2011). Le tableau 2 et la figure 7 (planche II) rendent compte de la progression de l'urbanisation sur la commune de La Faute-sur-Mer.

En 2010, La Faute-sur-Mer comptait 2210 parcelles urbanisées. Du village initial (quelque 300 parcelles construites en 1950), l'évolution a donc été fulgurante : multiplication par sept du bâti en 60 ans. 


\begin{tabular}{|c|c|c|c|c|c|c|c|}
\cline { 2 - 9 } \multicolumn{1}{c|}{} & $\begin{array}{c}\text { Nombre de } \\
\text { parcelles } \\
\text { construites } \\
\text { par période } \\
\text { (commune } \\
\text { entière) }\end{array}$ & $\begin{array}{c}\text { Nombre de } \\
\text { parcelles } \\
\text { construites } \\
\text { en zone inon- } \\
\text { dable par } \\
\text { période }\end{array}$ & $\begin{array}{c}\text { Part des par- } \\
\text { celles urbani- } \\
\text { sées situées } \\
\text { en ZI }\end{array}$ & $\begin{array}{c}\text { Nombre de } \\
\text { parcelles ur- } \\
\text { banisées dans } \\
\text { la commune } \\
\text { (cumul) }\end{array}$ & $\begin{array}{c}\text { Nombre de } \\
\text { parcelles ur- } \\
\text { banisées en ZI } \\
\text { (cumul) }\end{array}$ & $\begin{array}{c}\text { Nombre de } \\
\text { parcelles } \\
\text { construites } \\
\text { dans ZI }>1 \mathrm{~m} \\
\text { d'eau }\end{array}$ & $\begin{array}{c}\% \text { moins de } \\
1 \mathrm{~m}\end{array}$ \\
\hline avant 1950 & 331 & 76 & 23,0 & 331 & 76 & 4 & 5,3 \\
\hline $1950-1980$ & 822 & 432 & 52,6 & 1153,0 & 508,0 & 151 & 35,0 \\
\hline $1980-1990$ & 399 & 279 & 69,9 & 1552,0 & 787,0 & 88 & 31,5 \\
\hline $1990-2000$ & 409 & 266 & 65,0 & 1961,0 & 1053,0 & 84 & 31,6 \\
\hline $2000-2010$ & 247 & 174 & 70,4 & 2208,0 & 1227,0 & 133 & 76,4 \\
\hline $\begin{array}{c}\text { Cumul ou } \\
\text { moyenne }\end{array}$ & 2208 & 1227 & 55,6 & & & 460 & 37,5 \\
\hline
\end{tabular}

Tableau 2 : Évolution de la construction en zone inondable à La Faute-sur-Mer (source : DDTM85, DGI) Number of buildings in the flood prone area in La-Faute-sur-Mer

Après la création de la commune en 1953, l'implantation des services publics essentiels (bureau de poste, mairie) amorce la création de petits lotissements qui ne s’inscrivent pas dans un ensemble cohérent mais plutôt disséminés ça et là (Cabanne, 1977). Les premiers lotissements entourent le centre ancien puis s'étendent déjà dans les années 1970 vers les cordons dunaires au sud. Le fait d'urbaniser vers le sud du territoire communal répond à une logique topographique. L'urbanisation se fait entre les dunes forestières protégées de la Pointe d'Arçay et les zones basses marécageuses au bord du Lay. Cela signifie que le risque c'est-à-dire le caractère inondable des zones bordières de l'estuaire du Lay était connu et évité dans un premier temps par l'urbanisation. Cependant, cette urbanisation précoce vers le sud crée un espace vide qui sera comblé progressivement dans les années 1980-2010. D'ailleurs le nombre de bâtiments construits dans la zone inondée par Xynthia augmente au fil des décennies (tableau 2). En 1950, seules $23 \%$ des habitations construites alors étaient dans le périmètre inondé en 2010 dont 4 seulement ont reçu plus d'un mètre d'eau. $70 \%$ des parcelles construites à La Faute-sur-Mer dans la décennie 1980-1990 l'ont été en zone inondée 2010. Enfin, lors de la dernière décennie, sur 274 maisons construites, 174 le sont en zone inondée et 133 soit près de la moitié ont reçu plus d'un mètre d'eau le 28 février 2010.

On voit donc clairement que si le risque n'était pas pris en compte, il était pourtant implicitement connu puisque l'urbanisation s'est faite (en partie) en relation inverse du danger. Les parcelles les plus sûres (les plus hautes et les moins humides) ont été urbanisées d'abord puis progressivement les parcelles de plus en plus basses. Aucune des personnes décédées à La Faute le 28 février 2010 n'habitait une maison construite avant 1950. En revanche, neuf personnes (soit près du tiers des victimes) ont péri dans le lotissement des Doris. Cet espace, au point le plus bas du village (moins de $2 \mathrm{~m}$ NGF), près de l'exutoire d'un petit étier affluent du Lay, fut le dernier loti. Les dernières habitations étaient en voie d'achèvement en 2010 (figure 8).

L'absence ou l'inefficacité de la réglementation de l'usage du sol a déjà été démontrée. Renard (1984) note que dans de nombreuses communes littorales les Plans d'Occupation des Sols (POS) sont plus « un instrument de distribution des droits à construire que des documents d'aménagement ». On assiste en réalité à une véritable prédation où les acteurs fonciers et immobiliers locaux se partagent la manne foncière.

\begin{tabular}{|c|c|c|c||c|c|}
\cline { 2 - 5 } \multicolumn{1}{c|}{} & PPR approuvé & PPR prescrit & Pas de PPR & PCS & DICRIM \\
\hline $\begin{array}{c}\text { Nombre de communes } \\
\text { (44 au total })\end{array}$ & 19 & 5 & 20 & 0 & 0 \\
\hline
\end{tabular}

Tableau 3 : Prévention du risque de submersion marine (PPR) dans les communes littorales de sud-Vendée et de Charente-Maritime en février 2010 (Source : base GASPAR; Préfecture de Charente-Maritime; Préfecture de Vendée; MEEDDM, 2010)

Prevention of coastal flooding in coastal cities on February 2010 
La résistance de certains édiles à toute tentative de maîtrise d'occupation des sols par l'État a d'ailleurs été pointée par les retours d'expérience «Xynthia ».

Les plans de prévention des risques (PPR) ont été adoptés en 2002 et 2004 en Charente-Maritime mais une nouvelle fois La Faute-sur-Mer et L'Aiguillonsur-Mer font figure d'exception puisque le PPR (estuaire du Lay) était en application par anticipation seulement depuis 2007 avec une portée ô combien limitée puisque les espaces désignés en rouge sur ce PPR étaient ceux-là même qui étaient en voie de lotissement en 2010 et qui ont été submergés par plus de deux mètres d'eau (lotissement des Doris voir figure 7 - planche II).

Sur l'ensemble de ces 44 communes côtières situées entre le sud-Vendée (La Tranche-sur-Mer) et la Tremblade (17), la population est passée de 62000 habitants en 1821 à 100000 en 1946 et 182000 en 2007 (figure 2).

De plus, le type d'habitat produit lors de cette période de croissance urbaine était peu adapté au risque inondation.

\section{Des enjeux plus vulnérables}

La nature du bâti peut en effet jouer un rôle essentiel sinon dans la sauvegarde des biens matériels tout au moins dans celle des vies humaines (Jonkman et Kelman, 2005). Afin d'évaluer la dangerosité du bâti, nous en avons établi une typologie simplifiée en trois classes (figure 9) en considérant avant tout la

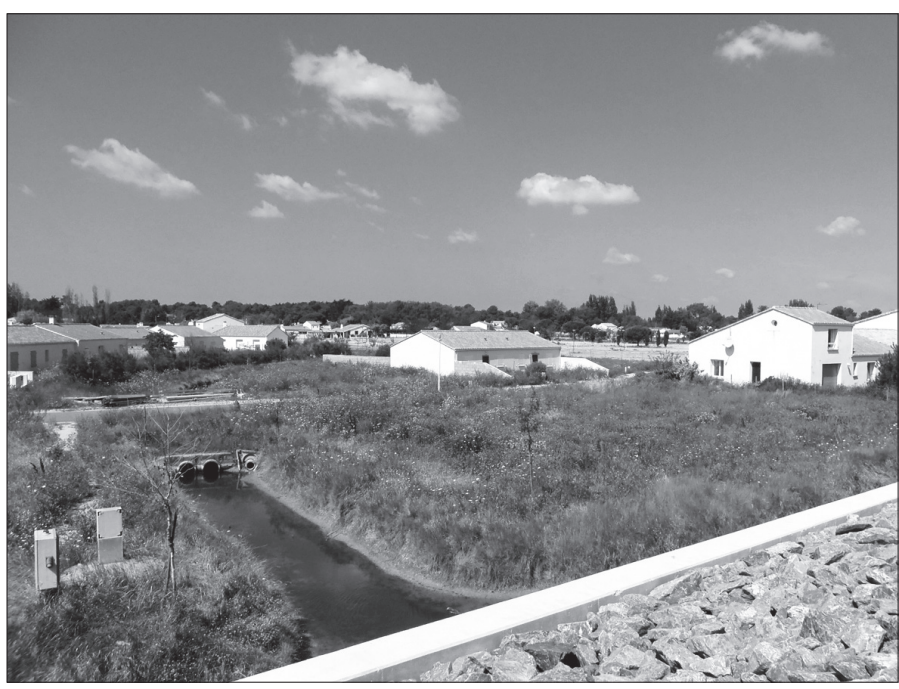

Figure 8 : «Cuvette de la Mort » à La Faute-sur-Mer vue depuis la digue du Lay. On voit au premier plan l'étier qui draine cet espace situé à environ $2 \mathrm{~m}$ NGF. On remarque également la nette prédominance des maisons basses de plain-pied (cliché : F. Vinet).

Low hing coastal zone called "Bowl of Death" at "La Faute-surMer" view from the Lay River's dike.

sauvegarde des vies humaines. La dangerosité a été évaluée par des observations de terrains. La première catégorie de «plain-pied » représente des habitations inadaptées pour face à des événements naturels de grande ampleur comme Xynthia. Lors de ces événements, des laisses de crue posées sur le toit attestent de la submersion totale de l'habitation. Ce type de bâti est par conséquent celui qui entraîne une plus grande vulnérabilité des occupants d'autant plus que sans sortie sur le toit, les habitants se trouvent litté-

Plus vulnérable

Moins vulnérable

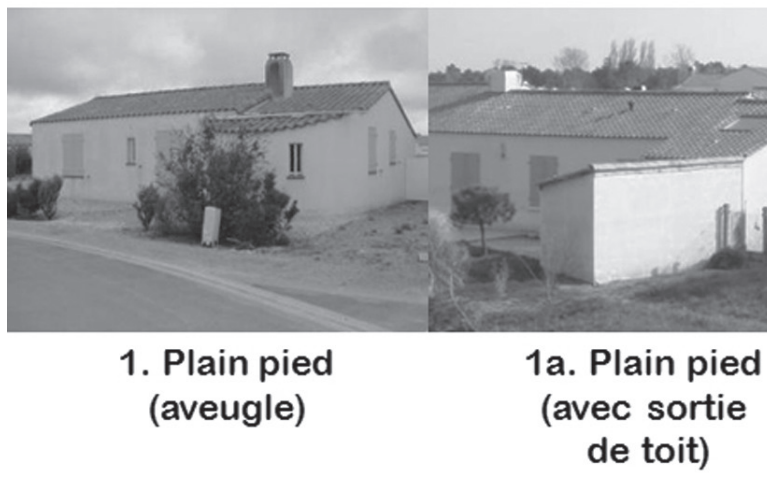

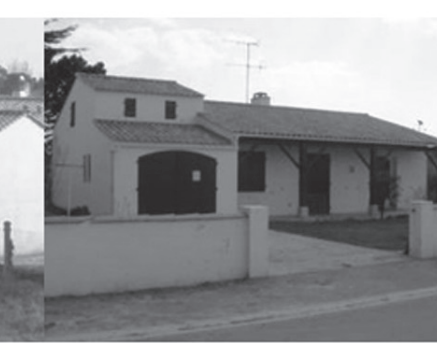

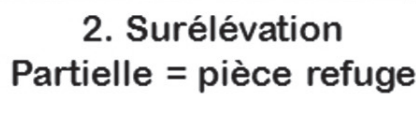

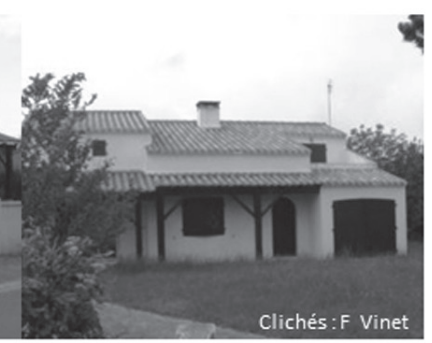

3. Au moins un étage complet

Figure 9 : Typologie simplifiée de la vulnérabilité du bâti à La Faute-sur-Mer Typology of houses in regard with their vulnerability facing flooding 
ralement piégés. Le type la présente une dangerosité presque égale mais l'ouverture sur le toit laisse une chance de survie aux résidents qui peuvent s'extraire de l'habitation et attendre les secours. La surélévation partielle ou pièce refuge (type 2) caractérise un bâti moins dangereux puisqu'elle permet aux populations de trouver refuge en cas de montée des eaux, cependant l'accès par l'intérieur n'est pas garanti et ne peut pas toujours abriter tous les occupants. Au contraire, les maisons à étages (type 3) donnent l'avantage d'accueillir tous les occupants de l'habitation.

Un travail d'identification du type de bâti a été effectué sur la zone noire initiale à La Faute-surMer. Sur les 676 maisons répertoriées, 444 sont de plain-pied soit $65 \%$. A contrario, seules $6 \%$ des habitations présentent un ou plusieurs étages. Ainsi, sur l'ensemble des deux départements, 32 sur les 41 personnes décédées suite à la submersion marine «Xynthia » sont décédées dans des maisons de plainpied (Vinet et al., 2011).

Or les règles d'urbanisme en vigueur dans les communes littorales et les besoins immobiliers exprimés par les populations ont favorisé la prolifération des maisons de plain-pied. L'exemple du plan d'occupation des sols de La Faute-sur-Mer est à cet égard édifiant. Le POS date de 1984 et a subi une dernière révision en 2000 avant d'être annulé en 2005 suite à une procédure judiciaire. Le rapport des inspections générales (MEEDDM, 2010) note qu'en 2010 le POS n'avait toujours pas été officiellement transformé en PLU comme l'exige la loi SRU. Les règles de constructibilité sont assez draconniènnes. Dans la zone la plus inondée, classée en NA (zone d'urbanisation future!), le règlement interdit les constructions d'une hauteur supérieure à $6 \mathrm{~m}$ au pignon. Le talutage est interdit. Les maisons ne sont pas construites sur vide sanitaire alors que les sols sont relativement humides en hiver. En outre, très peu d'habitations sont équipées d'ouverture de toit mais nous n'avons pas trouvé trace de l'interdiction formelle d'en installer.

L'objectif de ces règles était de préserver le cachet esthétique de la maison basse maraichine érigée en modèle résidentiel et touristique. D’ailleurs, la clientèle recherche ce type de maison. La taille moyenne des foyers résidant à La Faute-sur-Mer est passée de 2,7 personnes en moyenne en 1968 à moins de deux personnes actuellement, de 2,6 à 2 à Saint-Georgesd'Oléron ou de 3 à 2,2 à La Flotte-en-Ré. Outre la taille réduite des foyers, l'âge des personnes s'instal- lant en bord de mer ne plaide pas en faveur de maisons de grande taille ni $a$ fortiori d'habitations équipées d'escaliers. Ces habitations sont souvent d'abord construites ou acquises en résidence secondaire puis occupées en permanence au moment de la retraite. Le coût du foncier et de la construction et la faible solvabilité de la clientèle, pour la plupart régionale, ne permettraient pas d'envisager la construction de maisons à étages. Ainsi de nombreux facteurs pèsent en faveur de la diffusion de la maison de plain-pied, hérésie préventive au regard du risque inondation.

La figure 10 tente de résumer le processus de production du risque qui a transformé en une quarantaine d'années les espaces littoraux inondables en espaces fortement urbanisés. Face à des événements majeurs assez rares, les processus de gestion du risque et de préparation à la gestion de crise ont été défaillants. Si pour certaines communes, notamment en Charente-Maritime, ce schéma doit être nuancé, la commune de La Faute-sur-Mer représente un parangon de laxisme. Outre la maîtrise de l'occupation défaillante, la commune ne s'est guère préoccupée de planification de gestion de crise. La commune n'était pas dotée de Plan communal de sauvegarde. Elle ne disposait pas non plus de DICRIM concernant le risque de submersion marine.

La comparaison des temps longs de l'évolution des milieux et du temps court de l'urbanisation (figure 2) a bien montré que cette dernière avait " profité » d'une absence de tempête entre 1957 et les années 2000. Ainsi l'on a bien un système de production du risque dont la tempête Xynthia et ses dommages ont été l'épilogue... momentané. Reste à savoir si la catastrophe, non pas en tant qu'aboutissement d'un système de production du risque mais comme rupture dans les modes de gestion du risque, donnera un coup d'arrêt à ce processus où si la dialectique production du risque - catastrophe - oubli - production $d u$ risque - catastrophe va se perpétuer.

\section{Conclusion}

La catastrophe fait sens. Evénement brutal et « inattendu », elle prend des formes qui sont hors de nos schémas de représentation habituels. Elle révèle aussi des tendances cachées dans les processus de production du risque. La catastrophe vient de la rencontre entre des événements naturels de basse fréquence et forte intensité avec des enjeux vulné- 


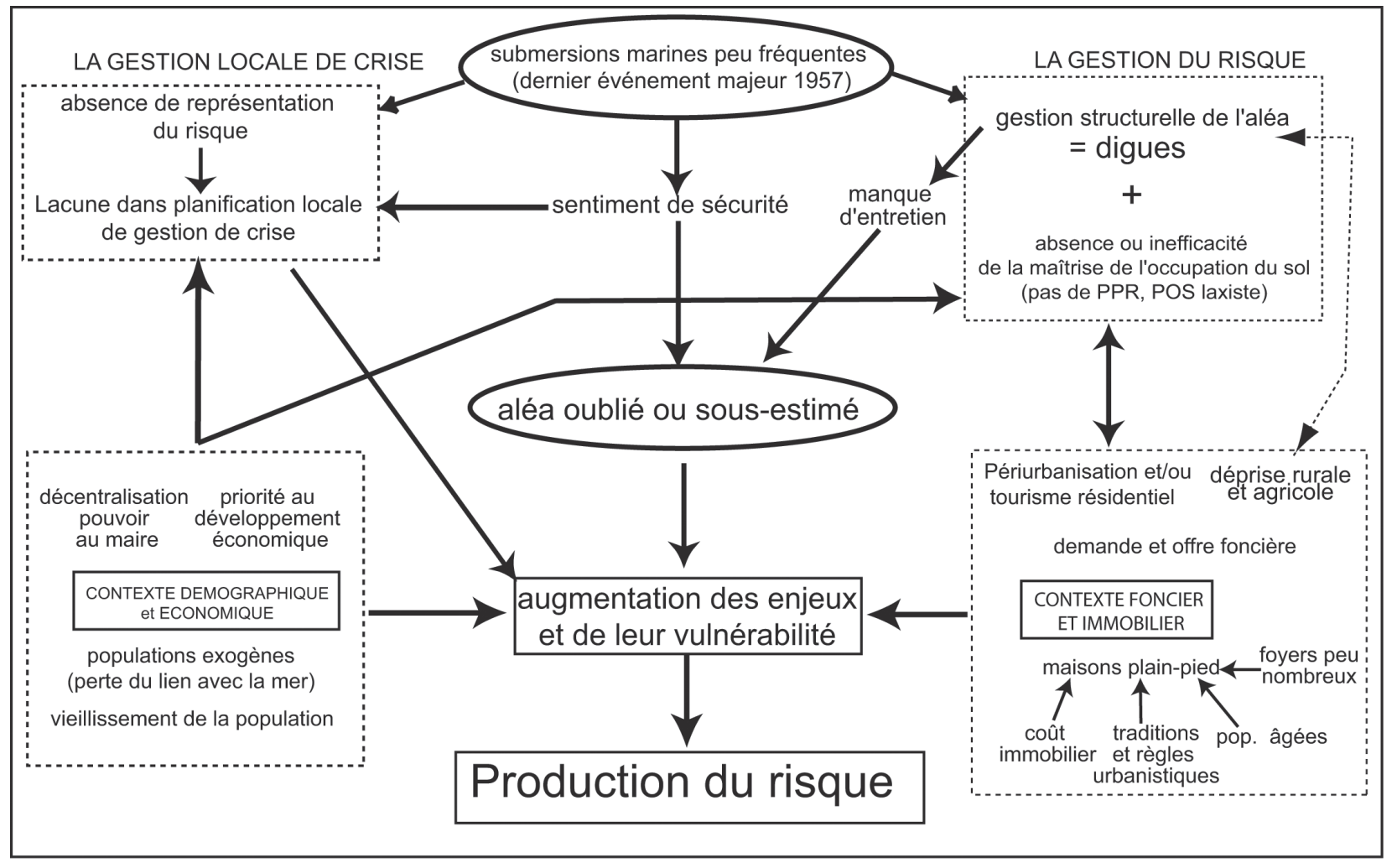

Figure 10 : Le processus de production du risque «submersion marine » dans les pertuis Charentais (1950-2010) The production of coastal flooding risk in the region hit by Xynthia storm

rables. Déclencheur de la crise, l'événement naturel paroxystique laisse une grande part de responsabilités aux facteurs humains. La submersion marine du 28 février 2010 a touché un territoire fragilisé et en mutation tant par des facteurs naturels (érosion, accumulation, déplacement de la flèche littorale) qu'anthropiques (croissance démographique, extension de l'urbanisation, etc.). Finalement, le processus de production du risque résulte d'une implantation humaine vulnérable sur un espace littoral fragile et fortement exposé, de par sa genèse même, au risque de submersion marine.

Or la production de ces enjeux vulnérables est sur la côte atlantique un phénomène très récent dans l'échelle du temps de retour des tempêtes majeures. La période d'accumulation de ces enjeux donc de production du risque a été trop courte pour que des événements majeurs viennent raviver la mémoire. S'ajoute l'arrivée de populations et d'activités peu liées à la mer ou en tout cas ne fréquentant la mer qu'en période estivale lorsque cette dernière est peu «hostile».
Pour assurer la prévention de telles catastrophes, la démocratie locale atteint ses limites. La trop grande proximité entre les décisions politiques et les enjeux fonciers et immobiliers n'est pas apte à offrir des conditions favorables à la maîtrise de l'occupation des sols. Ceci légitime l'intervention d'un acteur tel que l'État, plus éloigné des intérêts économiques locaux et chargé d'assurer la sécurité des citoyens. Cette analyse montre également que les catastrophes sont, sur le long terme, largement prévisibles spatialement à défaut d'être prédictibles dans le temps. De nombreux territoires en France qui ont subi des urbanisations inconsidérées en zone dangereuse sont ainsi vouées à subir ce type de catastrophe.

Enfin, le manque d'anticipation et de préparation au niveau local a été pointé du doigt. Malgré la connaissance vernaculaire de la submersion marine (les vimers), malgré les quelques études des services techniques compétents (Raison, 2008), il n'existait pas de représentation mentale des conséquences possibles de ces submersions. Entre la connaissance du risque par quelques experts ou services de l'État et la 
représentation mentale collective de la catastrophe, il reste de grands progrès à faire pour mettre la catastrophe dans le champ du possible.

\section{Bibliographie}

Allard J., Bertin X., Chaumillon E., Pouget F., 2008. Sand spit rhythmic development : A potential record of wave climate variations? Arçay Spit, western coast of France, Marine Geology, vol. 253, n³-4, p. 107-131.

ANDRÉ X., 1986. Élaboration et analyse de cartes bathymétriques détaillées du proche plateau-vendéo-charentais (golfe de Gascogne). Reconstitution des paléo-rivages de la transgression holocène, Thèse de doctorat, Université de Bordeaux 1, $274 \mathrm{p}$.

ANZIANi A., 2010. Xynthia : les leçons d'une catastrophe (rapport d'étape). Rapport d'information de M. Alain Anziani, fait au nom de la mission commune d'information sur les conséquences de la tempête Xynthia, nº 554 (2009-2010), 10 juin 2010.

Bounier A., 1957. Aspects morphologiques de la partie occidentale du Marais Poitevin, Norois, n ${ }^{\circ}$ 14, p. 175-207.

Cabanne C., 1977. Lévolution récente de L’Aiguillon-La Fautesur-Mer (Vendée), Norois, n 95, p. 37-55.

Chadenas C., Pottier P., Mercier D., Chauveau E., PouRINet L., 2011. Le prix d'une urbanisation abusive, Place publique, Hors-série, p. 19-24.

Chauveau E., Chadenas C., Comentale B., Pourinet L., 2011. Une tempête banale?, Place publique, Hors-série, p. 25-30.

Garnier E., Surville, F (dir.), 2010. La tempête Xynthia face à l'histoire. Submersions et tsunamis sur les littoraux français $d u$ Moyen Âge à nos jours, Paris, Le Croît Vif, 176 p.

Gouleau D., Jounnneau J.-M., Weber O., Sauriau P.-G., 2000. Short and long term sedimentation on MontportailBrouage intertidal mudflat, Marennes-Oleron Bay (France). Continental Shelf Research, vol. 20, n 12-13, p. 1513-1530.

Gruet Y., Sauriau P.-G., 1994. Paléoenvironnements holocènes du marais poitevin (littoral atlantique, France) : reconstitution d'après les peuplements malacologiques, Quaternaire, vol. $5, \mathrm{n}^{\circ} 2$, p. 85-94.

Jonkman S. N., Kelman I., 2005. An analysis of causes and circumstances of flood disaster deaths, Disasters, vol. 29, $\mathrm{n}^{\circ} 1$, p. $75-97$.
MEEDDM, 2010. Tempête Xynthia : retour d'expérience, évaluation et propositions d'actions, ministère de l'Écologie, de l'Énergie, du Développement Durable, Paris, 191 p.

Musereau, J., 2009. Approche de la gestion des cordons littoraux : mise au point et application d'un indice d'érosion (zone des Pertuis charentais, France), Thèse de doctorat, Université de Rennes 2, 305 p.

Pawlowski A., 1998. Géographie historique des côtes charentaises, Paris, Le Croît Vif, 235 p.

Pedreros R., Garcin M., Krien Y, Monfort Climent D., Mugica J., François B., 2010. Tempête Xynthia : compte rendu de mission préliminaire, Rapport BRGM/RP58261-FR, 45 p.

Raison S., 2008. Le classement des digues littorales au titre de la sécurité civile: un exemple de mise en ceuvre en Vendée, $\mathrm{X}^{\mathrm{e}}$ Journées Nationales Génie Côtier - Génie Civil, SophiaAntipolis, 14-16 octobre 2008, Gaufres P., Levacher D. et le Centre Français du Littoral (dir.), Tome 1, p. 283-292 [http://www.paralia.fr/jngcgc/10_27_raison.pdf].

Regrain R., 1980. Géographie physique et télédétection des marais charentais, Dolus d'Oléron, 512 p.

REnard J., 1972. Tourisme balnéaire et structures foncières : l'exemple du littoral vendéen, Norois, n 73, p. 67-79.

Renard J., 1976. Réflexions à propos de l'aménagement du littoral entre Loire et Gironde, Norois, n 92, p. 661-674.

RENARD J., 1984. Le tourisme : agent conflictuel de l'utilisation de l'espace littoral en France, Norois, n 121, p. 45-61.

Rousseaux F., 2009. Une méthode d'analyse pour mesurer l'impact des documents d'urbanisme sur la maîtrise de l'étalement urbain : l'exemple de La Rochelle, France, VertigO, vol. 9, n 2. [http://vertigo.revues.org/8682].

Verger F., 2009. Les zones humides du littoral français, Belin, Paris, $447 \mathrm{p}$.

Verger F., 2010. À propos des inondations récentes de la région de L'Aiguillon-sur-Mer, en Vendée, EchoGéo, [http://echogeo.revues.org/11890].

Vinet F, Defossez S., Leclere J.-R., 2011. Comment se construit une catastrophe?, Place publique, Hors-série, p. 9-19.

Weber N., 2004. Morphologie, Architecture des dépôts, évolution séculaire et millénaire du littoral charentais. Apports de la sismique réflexion combinée à des suivis bathymétriques et validés par des vibrocarottages, Thèse de Doctorat de l'Université de La Rochelle, 383 p. 